\title{
Evaluation of Coated Al Alloy Using the Breakpoint Frequency Method
}

\author{
Zhicao Feng*, G. S. Frankel*, \\ *Fontana Corrosion Center \\ The Ohio State University, Columbus, Ohio 43210-1185, USA
}

\begin{abstract}
Electrochemical Impedance Spectroscopy (EIS) was used to study coated Al alloys during long-term exposure in $5 \mathrm{wt} \% \mathrm{NaCl}$ solution. The breakpoint frequency extracted from Bode plots allows the determination of the electrochemically active area of the sample. It shifts from low to high frequency with increasing exposure time due to the enlargement of the active area. The appearance of a high breakpoint frequency $f_{h}$, usually in the range of $10^{2}$ to $10^{3} \mathrm{~Hz}$, occurred after a certain exposure time associated with a sharp drop of open circuit potential and low frequency impedance, and was accompanied by the visual initiation of corrosion under the coating. The incubation time before $f_{h}$ appears can be used as a criterion to evaluate the performance of chromate primer combined with different surface pretreatments. Optical profilometry analysis was conducted to determine the corroded area of coated sample after longterm exposure. The corroded area of one coating system was very close to, but that of another coating system was much higher than, the electrochemically active area estimated by the breakpoint frequency method, due to the different extent of attack. This indicates that the breakpoint frequency may be only valid for the determination of defect area during the initiation of attack, but probably not for the propagation to large regions of attack.
\end{abstract}

Key words: Al Alloys; Organic Coatings; Electrochemical Impedance Spectroscopy; Breakpoint Frequency

\section{Introduction}


High strength aluminum alloys are widely used in structural aircraft applications because of the combination of good mechanical properties and light weight. However, during exposure to corrosive environments, Al alloys are prone to localized corrosion such as pitting, intergranular and crevice corrosion [1-4]. Therefore, Al alloys for aircraft applications are usually protected from the environment using multi-layered organic coating systems $[5,6]$. The most aggressive environments for coated metals involve either immersion in an aqueous environment or exposure to wet conditions such as humid air, deposited aerosol droplets, and condensation. Unfortunately, no organic coating can behave as a perfect barrier to completely block water transport through them, due to their free volume and defects offering possible paths for water penetration where corrosion attack occurs [7, 8]. The active area of coatings can be used to indicate the coating quality and extent of degradation.

A good coating providing a large ohmic potential drop makes the metal substrate nonpolarizable, so that DC electrochemical methods like linear polarization are unable to evaluate the corrosion rate of coated metals [9]. Electrochemical impedance spectroscopy (EIS) is a technique capable of characterizing the electrochemical interface of coated metals and assessing corrosion resistant properties of coatings [10-13]. Fitting of EIS data to an equivalent circuit has been widely used to analyze EIS results for coated metals, providing important parameters such as coating capacitance, pore resistance in the coating, and metal/interface capacitance and resistance [14-20]. The metal/electrolyte interfacial capacitance measurement is one possible metric to quantify the electrochemically active area in the coating, under the assumption that the specific capacitance (capacitance per unit area) of the metal/electrolyte interface is a constant. In typical aqueous environments, metal/electrolyte interfacial specific capacitance is usually about 15 to $30 \mu \mathrm{F} / \mathrm{cm}^{2}[21,22]$. The electrochemically active area is equal 
to the measured metal/electrolyte interfacial capacitance divided by the specific capacitance [12]. Additionally, the change of pore resistance in the coating has been used by Haruyama et al. [23] to determine the electrochemically active area via the following expression:

$$
R_{p o}=\rho d / A_{d}
$$

where $R_{\text {po }}$ is the pore resistance in the coating. $A_{d}$ is the electrochemically active area. $\rho$ and $d$ are the specific bulk resistivity and coating thickness, respectively. The above approaches require computer modeling of the measured EIS spectra. Haruyama et al. proposed another method, the breakpoint frequency method, to determine electrochemically active area of the coating without modeling [23]. Based on that, Scully et al. proposed the low breakpoint frequency theory, which allows determination of the electrochemically active area ratio as small as $0.01 \%[11,12]$. More detailed information about breakpoint frequency is provided below.

The breakpoint frequency method has been utilized to evaluate the performance of organic coatings on steels [12-16, 19, 23, 24]. However, even though the corrosion performance of coated Al alloys is commonly assessed by EIS [25-27], the breakpoint frequency approach has rarely been used. Huang et al. [28] confirmed the excellent corrosion resistance of anodized Al alloy 6061 by noting that the breakpoint frequency was almost constant with exposure time. Instead of qualitatively confirming corrosion resistance, however, the breakpoint frequency method has potential beyond the qualitative confirmation of corrosion resistance as it is a powerful technique that can quantitatively calculate the active area of Al under coatings; monitor the early stage of coating degradation, and rank coating performance.

The purpose of this work is to evaluate the use of breakpoint frequencies to monitor the coating defect area with time for different coating systems on an Al alloy substrate. The appearance of a high breakpoint frequency is shown to be the time when the visually localized 
corrosion occurred. The defect areas estimated by the breakpoint frequency method are compared with that measured by optical profilometry after a long-term EIS test. The breakpoint frequency and low frequency impedance values are also used to rank the performance of different surface pretreatments under a chromate-containing primer.

\section{The Breakpoint Frequency Method}

The nested equivalent circuit shown in Fig. 1 is often used to model the response of coated metals. Scully $[10,12]$ utilized it to determine the correlation between the defect area ratio and breakpoint frequency based on the theory of Haruyama et al. [23]. The intact coating covering most of a metal can be considered to be a capacitor of magnitude $\mathrm{C}_{\mathrm{c}}$. Small defects in the coating such as pores are modeled by a resistor $\mathrm{R}_{\mathrm{p} .}$. Faradaic processes occurring at the electrolyte/metal interface at the end of the pores are modeled using a capacitor $C_{d}$ in parallel with a resistor $R_{d}$ representing the defect capacitance and polarization resistance, respectively. The synthesized Bode magnitude and phase plots shown in Fig. 2 were obtained using the nested simplified Randles circuit for a sample of area $10 \mathrm{~cm}^{2}$ and varying the defect area percentage from $0.00001 \%$ to $0.1 \%$. These curves were adapted from Scully's work $[10,12]$. Table 1 shows the defect area ratios and the corresponding values of each component assumed for the curves in Fig. 2. The solution resistance, $\mathrm{R}_{\mathrm{s}}$, was assumed to be $1 \Omega$ and $\mathrm{r}_{\mathrm{po}}$ was assumed to be $1 \Omega \mathrm{cm}^{2}$ because of the low resistivity of the solution in the coating pores [12]. Here, lower case letters represent area normalized values of each component. $c_{c}$ is typically on the order of 1 to $10 \mathrm{nF} / \mathrm{cm}^{2}$ [14], and was assigned here as $1 \mathrm{nF} / \mathrm{cm}^{2} . \mathrm{r}_{\mathrm{d}}$ was assumed to be $10^{3} \Omega \mathrm{cm}^{2}$ based on the charge transfer resistance of bare Al measured using EIS [29-31]. The double-layer capacitance per unit area is usually considered similar to that for mercury, 15 to $30 \mathrm{uF} / \mathrm{cm}^{2}$ [21, 22]. A linear relationship was found between double layer capacitance and corroded area for scratched and CPC-coated 
AA 2024-T3, exhibiting a double layer capacitance on the order of $10^{-4} \mathrm{~F} / \mathrm{cm}^{2}$ [32]. This was assigned to be the value of $c_{d}$ in Table 1 . The overlapped Bode magnitude curves in the high frequency range of Fig. 2 for all three defect area ratios reflect the constant values of $R_{s}$ and $C_{c}$. The two resistance plateaus and the capacitive region in between shift downward with increasing defect area ratio, which lowers $R_{p o}$ and $R_{d}$ (in $\Omega$ ) and increases $C_{d}$ (in F).

Breakpoint frequencies in the Bode magnitude plot are defined as a boundary where a capacitive region first transitions to a resistive region when the frequency shifts from high to low values $[10,12]$. At the transition, the impedance of the capacitance is equal to the plateau resistance and the phase angle is about $45^{\circ}$. So the breakpoint frequency can also be determined in the Bode phase plot where, following the curve from higher to lower frequency, the phase angle first shifts below $45^{\circ}[10,12]$. High and low breakpoint frequency were defined based on the feature of the Bode plots as follows [12]:

$$
\begin{aligned}
& f_{h}=\frac{1}{2 \pi \varepsilon \varepsilon_{o} \rho} \frac{A_{d}}{A} \\
& f_{l}=\frac{d}{2 \pi \varepsilon \varepsilon_{o} r_{d}} \frac{A_{d}}{A}
\end{aligned}
$$

where $f_{h}$ and $f_{l}$ are high and low breakpoint frequency, respectively, A and $\mathrm{A}_{\mathrm{d}}$ are the total exposed coating area and defect area, respectively, $\rho$ is the resistivity in pores, $d$ is the coating thickness, $\mathrm{r}_{\mathrm{d}}$ is the unit area defect resistance, and $\varepsilon$ and $\varepsilon_{\mathrm{o}}$ are the dielectric constant for the coating saturated with water and permittivity of free space, respectively. The relationship between $f_{h}$ and the defect area ratio is independent of the substrate. However, $f_{l}$ is dependent on the area normalized polarization resistance at the metal/electrolyte interface, indicating that the relationship between $f_{l}$ and the defect area ratio depends on the material. In this work, the 
substrate is always $\mathrm{Al}$ alloy. So, $f_{l}$ can still be used to evaluate coatings qualitatively. This area dependence of $f_{l}$ can only be observed for good coatings with a very small defect area.

The benefit of the breakpoint frequency method is its ability to monitor the defect area as a function of time only using the high frequency range of Bode magnitude plots, with no need for analyzing the complicated behavior in the low frequency range. Moreover, the double layer capacitance and charge transfer resistance of a specific bare metal are not needed when using the high breakpoint frequency method.

\section{Experimental}

The surfaces of the AA7075-T6 (UNS A97075) panel substrates were treated five different ways: chromate conversion coating (CCC, Alodine 1200S, Henkel Surface Technologies), Trivalent chromium pretreatment (TCP, SurTec 650 chromitAL, Surtec International), nonchromate pretreatment (NCP, Alodine 5700, Henkel Surface Technologies), adhesion promoter (AP, PreKote, Pantheon Industries), and clean only (CO) [33]. The CO sample was treated by degreasing and desmutting to remove organic contaminants, salt, lubricants, and metallic oxide particles that might have been embedded during rolling or machining. Two different primer coatings were painted on the pretreated panels: a chromate epoxy primer (PPG CA7233, MILPRF-23377 Type I, Class C2 coating [34]) and a Pr-rich primer (Deft 084, MIL-PRF-23377J Type I, Class N coating [34]). No topcoat was applied on top of the primer in this work. Two batches of coating systems were measured. The first batch of coated Al alloys had a chromate conversion coating $(\mathrm{CCC})$ with chromate primer or AP pretreatment with Pr-rich primer. The second batch of samples had the same chromate primer, but different surface pretreatments, including TCP, NCP, AP, and CO. 
A cylindrical PTFE cell with an inner surface area of $5.50 \mathrm{~cm}^{2}$ was positioned on the coated panel using silicone glue, which was allowed to dry for $24 \mathrm{~h}$. The cell was filled with naturallyaerated $5 \mathrm{wt} \% \mathrm{NaCl}$ solution. A graphite rod was used as the counter electrode and a Saturated Calomel Electrode (SCE) was the reference electrode. EIS measurements were carried out using a Gamry Instruments Reference 600 potentiostat. The impedance was collected in the frequency range from $10^{5}$ to $10^{-2} \mathrm{~Hz}$ with a $10 \mathrm{mV}$ voltage amplitude around the open circuit potential (OCP). 7 data points were collected per decade. EIS measurements were taken on the each coated Al alloy periodically (usually every day) for exposures of >100 days. The OCP was monitored for 15 min before each EIS measurement. Because of limited samples and the long period of the experiments, only one panel with each coating system was examined at each condition. The experimental reproducibility not investigated in this work.

After the long-term EIS measurement, the coatings were stripped by immersing the samples into concentrated nitric acid $\left(\mathrm{HNO}_{3}\right)$ for $20 \mathrm{~min}$. This treatment also removed corrosion products that formed under the coating $[5,6]$. Topographic analysis of the stripped panel was then conducted using optical profilometry (OP, Veeco Contour GT-K). The topographic images produced by OP were analyzed using a software package (Vision64TM v5.30 from Bruker) to quantify the corroded area under coatings [33].

\section{Results}

Fig. 3 shows Bode magnitude and phase angle plots for Al alloy coated with CCC pretreatment and chromate primer. The impedance data were collected periodically during 187 days exposure to $5 \mathrm{wt} \% \mathrm{NaCl}$ solution. The low frequency resistance was higher than $10^{9} \Omega$ and the Bode magnitude plot was a straight line for the first 8 days, indicating that the coating blocked the electrode completely, separating the metal substrate and electrolyte. After 21 days of 
exposure, the electrolyte penetrated the coating through pores and reached the substrate, activating the resistances in the pores and at the metal/electrolyte interface. This caused the magnitude plot to bend a little in the low frequency range, exhibiting a lower breakpoint frequency $f_{l}$. With increasing time, the exposed metal area was enlarged due to corrosion and coating degradation, causing the low frequency resistance to decrease and $f_{l}$ to shift to higher values. After a certain exposure time, 125 days exposure in this case, the electrochemically active area of the coating achieved a threshold value when an impedance plateau appeared associated with pore resistance in the middle frequency range. At this point, the high breakpoint frequency $f_{h}$ appeared. At that time, two separate time constants in the Bode phase plot became obvious, as shown in Fig. 3b. Poor separation of time constants before 125 days prevented their identification. The similarities of the Bode plots in Fig. 3 and Fig. 2 supported the use of the breakpoint frequency method for evaluating the active areas of these samples.

Fig. 4a shows change of $f_{l}$ and $f_{h}$ with exposure time for $\mathrm{Al}$ alloy coated with $\mathrm{CCC}$ and chromate primer. They were extracted from each Bode phase plot. Scully [12] stated that the measurable maximum and minimum values for $f_{l}$ and $f_{h}$ are 10 and $100 \mathrm{~Hz}$, respectively, which are marked as the two horizontal lines shown in Fig. 4a. $f_{l}$ could not be measured before 21 days, because the coating blocked the sample. After 21 days, $f_{l}$ was measured and found to slightly increase with exposure time from 0.1 to $2 \mathrm{~Hz}$ until 119 days. The appearance of $f_{h}$ occurred at 121 days, corrresponding to a sharp decrease of both the OCP and the low frequency impedance, $\mathrm{Z}_{0.01 \mathrm{~Hz}}$, values as shown in Fig. $4 \mathrm{~b}$. This indicates that a significant defect initiated under the coating. With further increasing time, the value of $f_{h}$ increased and approched the largest frequency tested, about $10^{5} \mathrm{~Hz}$, indicating propagation of the attack. Before the appearance of $f_{h}$, the OCP values were stablize at about $-300 \mathrm{mV}$ SCE because the CCC pretreatment passivated 
the substrate. After the initiation of attack on the substrate, the OCP values decreased to about $800 \mathrm{mV}$ SCE, which is close to the OCP of bare AA 7075-T6 in this solution.

Based on the correlation between the breakpoint frequency and the active area ratio in Fig. 2, the largest breakpoint frequency reaching $10^{5} \mathrm{~Hz}$ in Fig. 4 a corresponds to the active area ratio in the range of $0.01 \%$ to $0.1 \%$. Only one visual corrosion site was observed on the sample after 182 days exposure to $5 \mathrm{wt} \% \mathrm{NaCl}$ solution as shown in Fig. 5a and 5b. Optical profilometry was utilized to obtain a topograpohic map of this site, as shown in Fig. 5c. The depth and projected area of the pit were about $-120 \mu \mathrm{m}$ and $0.67 \mathrm{~cm}^{2}$, respectively. Because the total exposed area was $5.5 \mathrm{~cm}^{2}$, the visual defect area ratio was around $0.12 \%$ after 182 days EIS measurements, which is slightly higher than the ratio range estimated from the breakpoint frequency method. The period of time before the appearance of $f_{h}$ can also be considered as a criterion to evaluate the susceptiblity to coating degradation.

For comparsion, EIS measurements were conducted on the coating system consisting of adhesion promoter pretreatment and Pr-rich primer. Fig. 6 shows the Bode magnitude and phase plots of this coating during 121 days exposure to $5 \mathrm{wt} \% \mathrm{NaCl}$ solution. The breakpoint frequency was also observed to shift to higher values with increasing exposure time. A high breakpoint frequency occurred after only 12 hours exposure, much quicker than for the CCC pretreatment and chromate primer system, which was about 125 days. This indicates that attack initiated faster under the Pr-rich primer than the chromate primer or that the Pr-rich primer had more intrinsic defects than the chromate primer, such as pinholes, free volume, or blister initiation sites. The breakpoint frequency reached the highest frequency of $10^{5} \mathrm{~Hz}$ after 70 days exposure, indicating the defect area ratio was at least higher than $0.1 \%$ after the propagation. The attack under the chromate primer was still in the incubation stage at this time. Fig. 7 shows the effect of exposure 
time on the breakpoint frequency, $\mathrm{OCP}$, and $\mathrm{Z}_{0.01 \mathrm{~Hz}}$. The breakpoint frequency quickly shifted to high values and reached a plateau close to the maximum frequency $10^{5} \mathrm{~Hz}$. This corresponded to the sharp drop of $\mathrm{Z}_{0.01 \mathrm{~Hz}}$ values at the early stage, ending up at about $10^{5} \mathrm{ohms}$. The OCP increased from -900 to $-650 \mathrm{mV}$ SCE, and then dropped and stablized around $-800 \mathrm{mV} \mathrm{SCE}$. The adhesion promoter pretreatment did not strongly passivate the substrate, causing the OCP values to reach the OCP of bare Al alloys after only a short exposure. Although the breakpoint frequency was over $10^{5} \mathrm{~Hz}$ after 70 days corresponding to over $0.1 \%$ defect area ratio, it is still interesting to characterize and quantify the corroded area of the coatings after exposure. Fig. 8 displays the corrosion mophology and the topographic image after 121 days of exposure. The corroded area and area ratio are $14.77 \mathrm{~cm}^{2}$ and $2.7 \%$, repectively. This ratio is one order of magnitude higher than that determined using $10^{5} \mathrm{~Hz}$ as a breakpoint frequency. Accurate usage of the breakpoint frequency method requires the defect area ratio to be less than $1 \%$, which allows derivation of Eq. (2). Therefore, for small cell area $\left(5.5 \mathrm{~cm}^{2}\right.$ in this work), the breakpoint frequency method may be only valid for the determination of defect area ratio during the initiation of attack but not for the propagation to large regions of attack.

Because the breakpoint frequency method was successful for comparison of the two coating systems described above, it was also uilized to evaluate the second batch of coated samples with different surface pretreatments including TCP, NCP, AP, and CO. All of the samples had the same chromate primer after the surface pretreatment. Fig. 9 shows Bode magnitude plots of samples with each pretreatment exposed to $5 \mathrm{wt} \% \mathrm{NaCl}$ solution for over 300 days. The low frequency resistance $\mathrm{Z}_{0.01 \mathrm{~Hz}}$ of all the samples decreased with exposure time due to water uptake and coating degradation. The resistance plateau at the medium frequency range was only observed on the sample with NCP pretreatment, indicating that the high breakpoint frequency 
was exhibited at longer times and possibly visual attack initiated on the sample. The samples with other pretreatments exhibited only the low breakpoint frequency. Fig. 10 shows the Bode phase plots of chromate primer coated $\mathrm{Al}$ alloys with each surface pretreatment. As indicated by the magnitude plot, the phase plot shows that the breakpoint frequency shifted to higher frequencies for all the samples with exposure time. $\mathrm{Z}_{0.01 \mathrm{~Hz}}$ and the breakpoint frequency values were extracted from the Bode magnitude and phase angle plots, respectively, and are shown in Fig. 11. The higher values of breakpoint frequency correspond to the lower values of $Z_{0.01 \mathrm{~Hz}}$, due to the larger defect area ratio of the coating. This indicates the performance rank of the chromate primer on the surface pretreatments is $\mathrm{AP}>\mathrm{CO} \approx \mathrm{TCP}>\mathrm{NCP}$.

Fig. 12 shows the surface morphology of samples with different surface pretreatments after over 300 days of exposure. The left and right side of each part are before and after the coating was stripped, respectively. Visual blisters were only exhibited by the coated sample with NCP pretreatment, as shown in the yellow rectangles in Fig. 12b. Slight corrosion stains were observed after removing the coating. OP was not able to distinguish the two corrosion sites from the surrounding uncorroded areas, due to the very strong roughness of the substrate. Image J, a photo analysis software, was used to quantify the corroded area from the optical images. The area was about $2.3 \mathrm{~mm}^{2}$ corresponding to a defect area ratio of about $0.4 \%$, which is two orders of magnitude higher than the expected area ratio estimated from the highest breakpoint frequency, about $5000 \mathrm{~Hz}$ in Fig. 11b. The chromate primer coated samples with TCP, CO, and AP pretreatments exhibited no obvious corrosion attack. From visual comparison, the performance rank-order of the surface pretreatments is $\mathrm{AP} \approx \mathrm{CO} \approx \mathrm{TCP}>\mathrm{NCP}$, which is almost consistent with the order from the breakpoint frequency and $\mathrm{Z}_{0.01 \mathrm{~Hz}}$ values, except the visual comparison is not able to distinguish the performance of AP, $\mathrm{CO}$, and TCP. 
TCP also passivated the substrate like CCC, causing the higher OCP values than other nonpassivated pretreatments including NCP, CO and AP, as shown in Fig. 13. The OCP values of non-passivating pretreatments were between $-750 \mathrm{mV}$ and $-900 \mathrm{mV} \mathrm{SCE}$, close to the value of the bare Al alloy. The TCP treatment increased OCP to around $-500 \mathrm{mV} \mathrm{SCE}$, which is lower than the value measured for the CCC pretreatment before significant corrosion occurred, about $300 \mathrm{mV}$ SCE, as seen in Fig. $4 \mathrm{~b}$.

\section{Discussion}

To understand how the pore resistance, metal/electrolyte resistance and capacitance change with expanding defect area, the observed spectra for samples with CCC pretreatment and chromate primer were fitted using the defective coating equivalent circuit. The fitted lines are shown in Fig. 14 and the corresponding fit values of each component are presented in Table 2. Since the spectra did not exhibit a high frequency limit and $R_{s}$ is independent of the defect area, it was fixed as $1 \Omega$ during the computer fitting. One of the assumptions for the breakpoint frequency method is that the defect area is less than $1 \%$ of total cell area, making $\mathrm{A}-\mathrm{A}_{\mathrm{d}}$ approximately equal to A [12]. As a result, the coating capacitance is also independent of the defect area and does not change with exposure time, as shown in Table 2. It should be noted that the coating capacitance in this case is the value after saturation of the coating with water, which occurred quickly, usually in the first 24 hours [14]. The visual defect area ratio of the chromate coating system was around $0.12 \%$ (less than $1 \%$ ) after 182 days EIS measurements, as shown in Fig. 5, which is in good agreement with the defect area ratio evaluated by the breakpoint frequency. For the Pr coating system as shown in Fig. 8, however, the attack area ratio after 121 days EIS measurements was $2.7 \%$. This is greater than the limit for the application of the breakpoint frequency method, which is $1 \%$ of total cell area. The attack area ratio is almost two 
order magnitudes higher than that estimated by the breakpoint frequency. This supports the notion that the breakpoint frequency is only valid for very small defect areas or for the initial stage of coating degradation, and not for the defect propagation to a large scale. The other assumption for the breakpoint frequency method is the constant unit area resistance and capacitance in defects, allowing the determination of defect area by measuring resistance and capacitance. Based on that, the measured resistance including $R_{p o}$ and $R_{d}$ should decrease with expanding defect area. However, the measured defect capacitance $\mathrm{C}_{\mathrm{d}}$ should increase with expanding defect area. Such a trend is seen in Table 2. In addition, the resistance and capacitance should change by one order of magnitude when the breakpoint frequency or the defect area increases by one order of magnitude. However, the data in Table 2 do not show this relationship. This might be caused by the complexity of the metal/electrolyte interface, which is not modeled well by a pure capacitor parallel with a resistor. Instead of a pure capacitor, a constant phase element is usually utilized to model the capacitive behavior of coatings [35, 36].

Organic coatings protect the metal substrate in different ways. The primary mechanism is to act as a physical barrier separating metal and corrosive electrolyte. This barrier provides a large ohmic potential drop making metal substrate hard to be polarized. For instance, multi-layered coatings on $\mathrm{Al}$ alloy panels can protect them from anodic polarization by adjacent noble material fasteners for a certain time $[5,6]$. However, water uptake occurs quickly, causing the ohmic potential drop to decrease due to the relatively low resistance of the solution in the pores of coatings. After water approaches the metal substrate, corrosion attack can happen at anodic and cathodic sites. The coating can still protect the metal substrate after water uptake by preventing formation of a bulk electrolyte at the coating/substrate interface and thus limiting the interaction between anodic and cathodic sites. The charge transfer reaction occurs easier on both cathodic 
and anodic sites in the presence of a bulk electrolyte. Even when a bulk electrolyte phase exists at the metal/coating interface, inhibitors such as soluble chromate and $\mathrm{Pr}$ ions can be released from the coatings, migrate to the corrosion sites and prevent further attack [37-39].

The coating protectiveness mentioned above can be estimated by the important electrical parameters in the equivalent circuit, including the low frequency resistance $\mathrm{Z}_{0.01 \mathrm{~Hz}}$ and the breakpoint frequency. The breakpoint frequencies in Fig. 11 indicate the coating protectiveness rank of the surface pretreatments is $\mathrm{AP}>\mathrm{CO} \approx \mathrm{TCP}>\mathrm{NCP}$. Higher protectiveness means fewer intrinsic defects such as pinholes, free volume, and blisters, or larger resistance to the propagation of such defects, or a combination of both. Coating degradation was initiated at these intrinsic defects, activating larger corrosion areas under the coating and causing the breakpoint frequency shift to large values. Therefore, it is valid to evaluate coating protectiveness by monitoring the breakpoint frequency. The same chromate primer coated Al alloys with the same surface pretreatments, but with artificial scribes, were exposed to ASTM B117 for 100 days in a previous study [33]. Two " $\mathrm{X}$ " patterns were manually scribed into the coated aluminum alloy panels making sure to expose fresh Al metal at the bottom of the scribes. The width, depth and length of each as-formed scribe were about $250 \mu \mathrm{m}, 20 \mu \mathrm{m}$ and $20 \mathrm{~mm}$, respectively. In that study [33], the corrosion attack of the sample with NCP pretreatment was worse than the samples with other pretreatments. The panel with AP pretreatment exhibited no obvious corrosion attack on both " $\mathrm{X}$ " scribes. The corrosion extent of samples with TCP and CO pretreatment was between the NCP and AP pretreatment. These observations are consistent with the trends of low frequency resistance $\mathrm{Z}_{0.01 \mathrm{~Hz}}$ and breakpoint frequency in Fig. 11, which were determined during immersion in chloride solution. These observations suggest that the propagation of attack at a scribe, which is an extrinsic defect, during exposure in the salt fog chamber is related to the 
propagation of intrinsic defects during immersion as indicated by EIS measurements. It is also interesting that the breakpoint frequency of TCP, $\mathrm{CO}$, and AP remained at low values less than $10 \mathrm{~Hz}$ for over 300 days in $\mathrm{NaCl}$ solution. They also maintained high $\mathrm{Z}_{0.01 \mathrm{~Hz}}$ values of more than $10^{7} \Omega \mathrm{cm}^{2}$ and exhibited no visual corrosion attack for over 300 days exposure. It should be noted that ASTM B117 is an extremely aggressive environment compared to a full immersion test owing to the greater exposure to oxygen [5]. The good performance of samples with scribes in ASTM B117 and during immersion in chloride solution is strong evidence of the good performance of chromate primer combined with the AP pretreatment in that environment.

The excellent performance of protective coatings makes it difficult to compare them or predict their lifetime in a short period of time. Bierwagen et al. [26] plotted the low frequency resistance of different coatings as a function of time and extrapolated their lifetime using the impedance of bare metal as a failure criterion. It was assumed that $\log \left(\mathrm{Z}_{0.01 \mathrm{~Hz}}\right)$ exhibits a linear relationship with exposure time until coating failure. This assumption is in conflict with at least two very important observations in this study. Firstly, $\log \left(\mathrm{Z}_{0.01 \mathrm{~Hz}}\right)$ values exhibited a sharp drop at the very early stage associated with water uptake and another drop when significant corrosion initiated, as shown in Fig. 4 b. Between those two times, $\log \left(\mathrm{Z}_{0.01 \mathrm{~Hz}}\right)$ was almost constant around 7 to 8 , probably because the coating limited the formation of bulk electrolyte phase at the metal/coating interface. Therefore, it may be inappropriate to model the change of $\log \left(\mathrm{Z}_{0.01 \mathrm{~Hz}}\right) \mathrm{vs}$. time using a linear relationship. Fig. 11a also shows a non-linear relationship between $\log \left(\mathrm{Z}_{0.01 \mathrm{~Hz}}\right)$ and exposure time, although the second resistance drop did not occur. Additionally, the impedance of bare metal might not be a relevant coating failure criterion. A 3\% visual corroded area (ASTM D 610 rating of 5) was defined arbitrarily as coating failure [10]. A correlation of threshold impedance and visual corroded area percentage has been developed on epoxy 
polymide-coated steel and Araldite-coated $\mathrm{Mg}$ alloy [10]. The threshold low frequency impedance was about $10^{6}$ to $10^{7} \Omega \mathrm{cm}^{2}$ for coated steel and about $4 \times 10^{8} \Omega \mathrm{cm}^{2}$ for coated $\mathrm{Mg}$ alloy, which are both much higher than the impedance of bare metal. Therefore, a linear extrapolation of low frequency resistance to the bare metal impedance would be much less conservative for prediction of coating lifetime. EIS actually is a more useful technique to characterize coated metals before visual attack to obtain information such as water uptake and water saturation, small defect area estimation, and bulk electrolyte formation at the metal/coating interface.

In the present work, the appearance of $f_{h}$ (over $10^{2} \mathrm{~Hz}$ ) corresponds to visual corrosion initiation. It is difficult to observe such small amounts of corrosion before coating removal. After that, the attack propagated causing the breakpoint frequency to shift to even higher frequency values. The critical $f_{h}$ of $10^{2} \mathrm{~Hz}$ could be considered as a criterion to assess the incubation time before visually localized corrosion under coatings to rank different coating systems. This method avoids fitting and analyzing complex impedance at very low frequency. However, excellent coating systems postpone the appearance of the high breakpoint frequency and increase the incubation time to over 300 days. This was the case for chromate primer coating on TCP, CO, and AP pretreatments, as shown in Fig. 11b. Therefore, to compare different coating systems, it may be necessary to shorten the period before the appearance of the high breakpoint frequency in an appropriate way. Pre-polarizing coated metal can accelerate coating degradation by speeding up either metal dissolution or cathodic reactions, because corrosion products, hydroxyl ions, and hydrogen ions can induce coating disbondment [10]. A test cycle involving a sequence of OCP, EIS, and potentiostatic hold has been used to monitor the significant decrease of low frequency impedance of coatings in a few cycles [40]. The same test protocol can also be used to observe 
the breakpoint frequency shift and then to characterize expansion of defect areas with the test cycle number. Another way to accelerate coating degradation may be galvanic coupling with a noble metal, which provides large cathodic current driving the metal dissolution, similar to anodic pre-polarization. A combination of Al alloy panel and noble metal fastener is also similar to structures used in real applications. A coated Al alloy panel couple with bare stainless steel 316 fasteners has been recently been studied to provide an accelerated response to lab and field environments $[5,6,33]$. A serial EIS measurement can be conducted on the coated area near the fasteners after pre-exposure. The fasteners may accelerate the coating degradation during preexposure and then EIS could be utilized to quantify the degraded area by monitoring the breakpoint frequency shift.

\section{Conclusions}

EIS measurements were performed over long time periods on chromate primer coated Al alloy panels with different surface pretreatments including $\mathrm{CCC}, \mathrm{TCP}, \mathrm{NCP}, \mathrm{CO}$ and AP pretreatments. The breakpoint frequency method was used to monitor the increase in defect area for different coating systems. The specific conclusions are as follows:

1. A correlation was obtained between the high breakpoint frequency and the electrochemically active area on coated Al alloys. OP was used to quantify the active areas after long-term EIS measurements, which were one or two orders of magnitude larger than the area estimated by the breakpoint frequency. This is evidence of the limitation of the breakpoint frequency method; it may be only valid for very small defect area of the coating or for the initial stage of coating degradation, but not to assess defect propagation to a large scale. 


\section{Acknowledgment}

This work was supported by Office of the Secretary of Defense Technical Corrosion Collaboration through the US Air Force Academy.

\section{References}

[1] R.G. Buchheit, R.P. Grant, P.F. Hlava, B. McKenzie, G.L. Zender, Journal of the Electrochemical Society 144 (1997) 2621-2628.

[2] R.G. Buchheit, M.A. Martinez, L.P. Montes, Journal of the Electrochemical Society 147 (2000) 119124.

[3] Q.J. Meng, G.S. Frankel, Journal of the Electrochemical Society 151 (2004) B271-B283.

[4] T.S. Huang, G.S. Frankel, Corrosion 63 (2007) 731-743.

[5] Z. Feng, G.S. Frankel, C.A. Matzdorf, Journal of the Electrochemical Society 161 (2014) C42-C49.

[6] Z. Feng, G.S. Frankel, Corrosion 70 (2014) 95-106.

[7] T. Nguyen, J.B. Hubbard, J.M. Pommersheim, Journal of Coatings Technology 68 (1996) 45-56.

[8] T. Nguyen, E. Byrd, D. Bentz, C.J. Lin, Progress in Organic Coatings 27 (1996) 181-193.

[9] A. Amirudin, D. Thierry, Progress in Organic Coatings 26 (1995) 1-28.

[10] J.R. Scully, S.T. Hensley, Corrosion 50 (1994) 705-716.

[11] J.R. Scully, Journal of the Electrochemical Society 136 (1989) 979-990.

[12] H.P. Hack, J.R. Scully, Journal of the Electrochemical Society 138 (1991) 33-40.

[13] M. Kendig, J. Scully, Corrosion 46 (1990) 22-29.

[14] F. Mansfeld, Journal of Applied Electrochemistry 25 (1995) 187-202. 
[15] F. Mansfeld, M.W. Kendig, S. Tsai, Corrosion 38 (1982) 570-580.

[16] F. Mansfeld, Corrosion 37 (1981) 301-307.

[17] F. Mansfeld, M.W. Kendig, S. Tsai, Corrosion 38 (1982) 478-485.

[18] F. Mansfeld, M.W. Kendig, Journal of the Electrochemical Society 135 (1988) 828-833.

[19] F. Mansfeld, Electrochimica Acta 35 (1990) 1533-1544.

[20] M. Kendig, F. Mansfeld, S. Tsai, Corrosion Science 23 (1983) 317-329.

[21] J. Lawrence, R. Parsons, R. Payne, Journal of Electroanalytical Chemistry 16 (1968) 193-\&.

[22] D.C. Grahame, B.A. Soderberg, Journal of Chemical Physics 22 (1954) 449-460.

[23] R. Hirayama, S. Haruyama, Corrosion 47 (1991) 952-958.

[24] C.H. Tsai, F. Mansfeld, Corrosion 49 (1993) 726-737.

[25] B.R. Hinderliter, S.G. Croll, D.E. Tallman, Q. Su, G.P. Bierwagen, Electrochimica Acta 51 (2006)

4505-4515.

[26] G. Bierwagen, D. Tallman, J.P. Li, L.Y. He, C. Jeffcoate, Progress in Organic Coatings 46 (2003)

148-157.

[27] A. Simoes, D. Battocchi, D. Tallman, G. Bierwagen, Progress in Organic Coatings 63 (2008) 260-

266.

[28] Y. Huang, H. Shih, H. Huang, S. Wu, S. Ramanathan, C. Chang, F. Mansfeld, Corrosion Science 50 (2008) 3569-3575.

[29] A.K. Mishra, R. Balasubramaniam, Corrosion Science 49 (2007) 1027-1044.

[30] N.R. Tailleart, R. Huang, T. Aburada, D.J. Horton, J.R. Scully, Corrosion Science 59 (2012) 238-248.

[31] R.G. Buchheit, M.D. Bode, G.E. Stoner, Corrosion 50 (1994) 205-214.

[32] F. Gui, R.G. Kelly, Electrochimica Acta 51 (2006) 1797-1805.

[33] Z. Feng, J. Boerstler, G.S. Frankel, C.A. Matzdorf, Corrosion 71 (2015) 771-783.

[34] MIL-PRF-23377K, "Performance Specification Primer Coatings: Epoxy, High Solids" (Lakehurst, NJ: Naval Air Warfare Center, Air Division, 2012).

[35] E. Frechette, C. Compere, E. Ghali, Corrosion Science 33 (1992) 1067-1081.

[36] C. Compere, E. Frechette, E. Ghali, Corrosion Science 34 (1993) 1259-1274.

[37] J. Zhao, L. Xia, A. Sehgal, D. Lu, R.L. McCreery, G.S. Frankel, Surface \& Coatings Technology 140 (2001) 51-57.

[38] J. Zhao, G. Frankel, R.L. McCreery, Journal of the Electrochemical Society 145 (1998) 2258-2264.

[39] W.G. Zhao, J.G. Wang, H.L. Zhao, J.Q. Hou, Q.C. Jiang, Journal of Alloys and Compounds 479

(2009) L30-L35.

[40] M. Bethencourt, F.J. Botana, M.J. Cano, R.M. Osuna, A. Marcos, Progress in Organic Coatings 49

(2004) 275-281 . 


\section{Table}

Table 1. Defect area ratio and values of each component assumed for curves in Fig. 2.

\begin{tabular}{c|ccc}
\hline \multirow{2}{*}{ Assumed values } & \multicolumn{3}{|c}{ Defect area ratio, $\mathrm{A}_{\text {total }}=10 \mathrm{~cm}^{2}$} \\
\cline { 2 - 3 } & $0.1 \%$ & $0.01 \%$ & $0.00001 \%$ \\
\hline $\mathrm{R}_{\mathrm{s}}=1 \Omega$ & $1 \Omega$ & $1 \Omega$ \\
\hline $\mathrm{C}_{\mathrm{c}}=10^{-9} \mathrm{~F} / \mathrm{cm}^{2} * \mathrm{~A}_{\mathrm{total}}$ & $10^{-8} \mathrm{~F}$ & $10^{-8} \mathrm{~F}$ & $10^{-8} \mathrm{~F}$ \\
\hline $\mathrm{R}_{\mathrm{po}}=1 \Omega \mathrm{cm}^{2} / \mathrm{A}_{\mathrm{d}}$ & $10^{2} \Omega$ & $10^{3} \Omega$ & \\
\hline $\mathrm{R}_{\mathrm{d}}=10^{3} \Omega \mathrm{cm}^{2} / \mathrm{A}_{\mathrm{d}}$ & $10^{5} \Omega$ & $10^{6} \Omega$ & $10^{-10} \mathrm{~F}$ \\
\hline $\mathrm{C}_{\mathrm{d}}=10^{-4} \mathrm{~F} / \mathrm{cm}^{2} * \mathrm{~A}_{\mathrm{d}}$ & $10^{-6} \mathrm{~F}$ & $10^{-7} \mathrm{~F}$ & \\
\hline
\end{tabular}


Table 2. Fitted values of each component in the equivalent circuit used for modeling the EIS spectra for CCC-treated and chromate primed AA7075-T6 that is shown in Fig. 14. Total cell area is $5.5 \mathrm{~cm}^{2}$.

\begin{tabular}{c|ccccc}
\hline Component & $\mathrm{f}_{\mathrm{l}}=10^{-1} \mathrm{~Hz}$ & $\mathrm{f}_{\mathrm{l}}=10^{0} \mathrm{~Hz}$ & $\mathrm{f}_{\mathrm{h}}=10^{2} \mathrm{~Hz}$ & $\mathrm{f}_{\mathrm{h}}=10^{3} \mathrm{~Hz}$ & $\mathrm{f}_{\mathrm{h}}=10^{4} \mathrm{~Hz}$ \\
& 21 days & 66 days & 121 days & 134 days & 162 days \\
\hline $\mathrm{R}_{\mathrm{s}} / \Omega$ & 1 & 1 & 1 & 1 & 1 \\
$\mathrm{C}_{\mathrm{c}} / \mathrm{F}$ & $1.4 \times 10^{-9}$ & $1.5 \times 10^{-9}$ & $1.5 \times 10^{-9}$ & $1.6 \times 10^{-9}$ & $1.5 \times 10^{-9}$ \\
$\mathrm{R}_{\mathrm{po}} / \Omega$ & $1.3 \times 10^{7}$ & $3.3 \times 10^{6}$ & $7.2 \times 10^{5}$ & $8.4 \times 10^{4}$ & $1.2 \times 10^{4}$ \\
$\mathrm{C}_{\mathrm{d}} / \mathrm{F}$ & $3.3 \times 10^{-9}$ & $4.6 \times 10^{-9}$ & $5.2 \times 10^{-8}$ & $9.3 \times 10^{-8}$ & $1.4 \times 10^{-6}$ \\
$\mathrm{R}_{\mathrm{d}} / \Omega$ & $2.4 \times 10^{8}$ & $4.0 \times 10^{7}$ & $5.8 \times 10^{6}$ & $2.9 \times 10^{6}$ & $4.4 \times 10^{4}$ \\
\hline
\end{tabular}




\section{Figures}

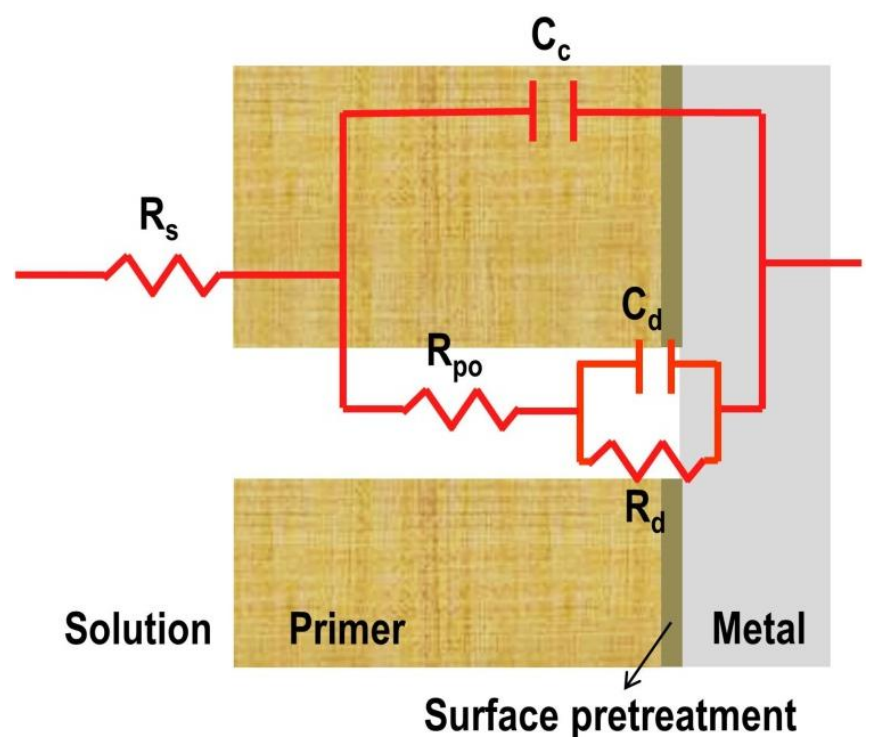

Fig. 1. Nested simplified Randles circuit of a coated Al alloy with a defect. $R_{s}$ is the solution resistance, $C_{c}$ is coating capacitance and $R_{p o}$ is pore resistance. $C_{d}$ and $R_{d}$ represent defect capacitance and resistance associated with reactions at the pore bottom. 

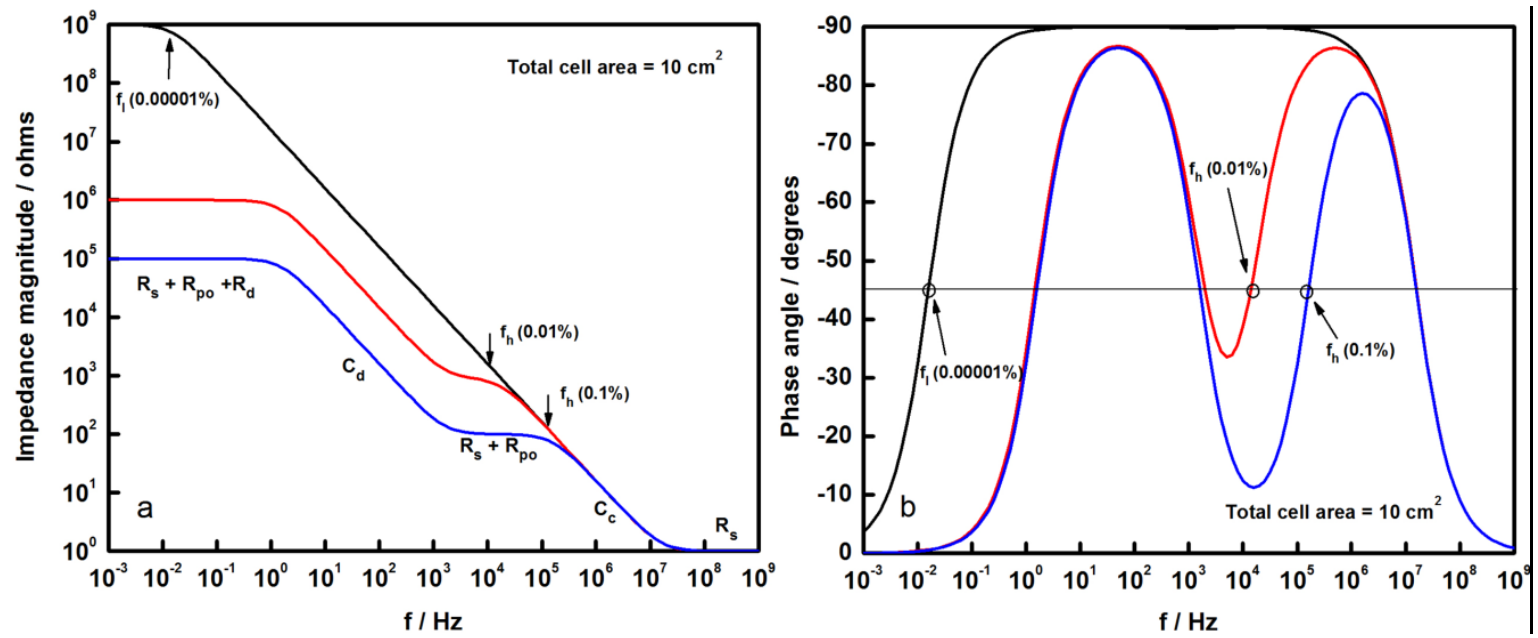

Fig. 2. Effect of defect area percentage on impedance magnitude and phase angle behavior of a coated Al equivalent circuit. Total sample area assumed is $10 \mathrm{~cm}^{2}$. Figure adapted from work of Scully $[10,12]$. 

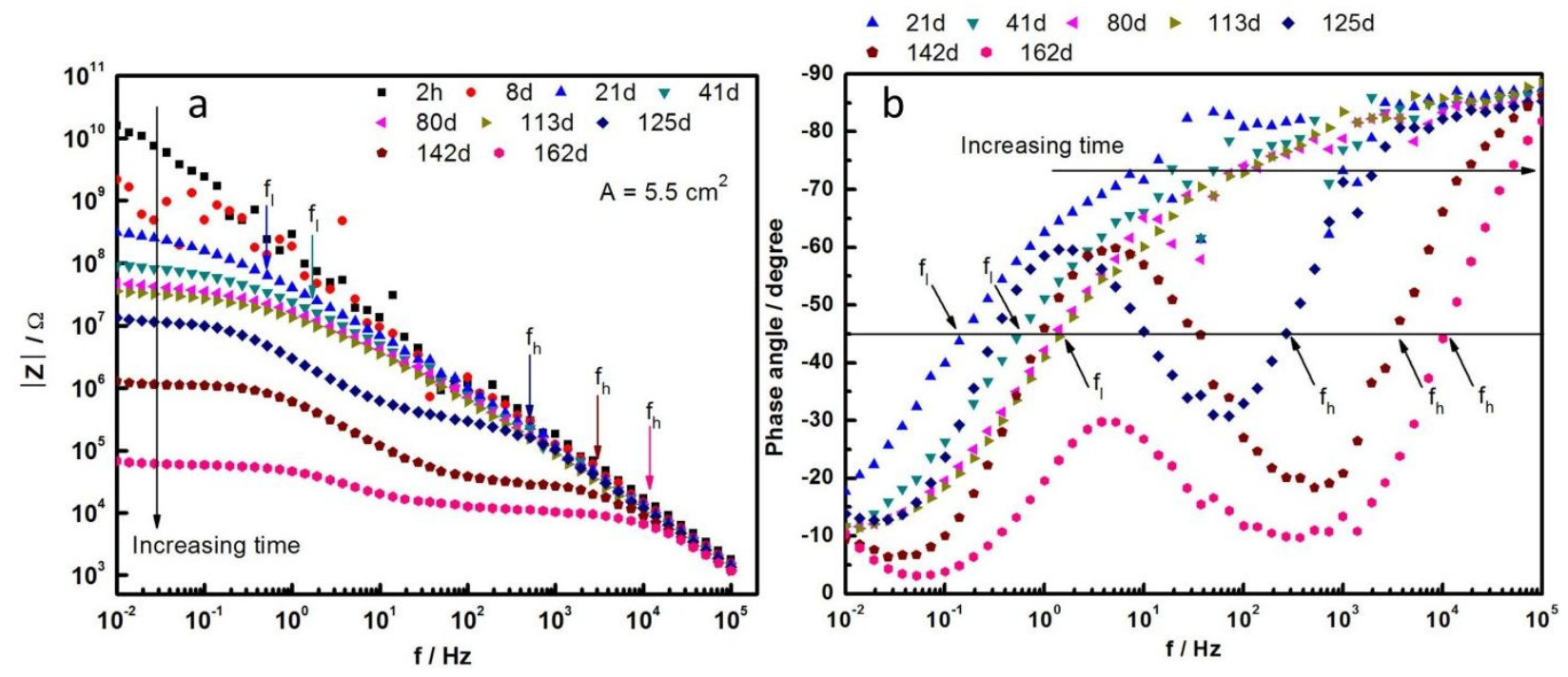

Fig. 3. Bode plots of Al alloy coated with CCC pretreatment and chromate primer. EIS measurments were performed periodically during 162 days exposure to $5 \mathrm{wt} \% \mathrm{NaCl}$ solution. Sample area is $5.5 \mathrm{~cm}^{2}$. 

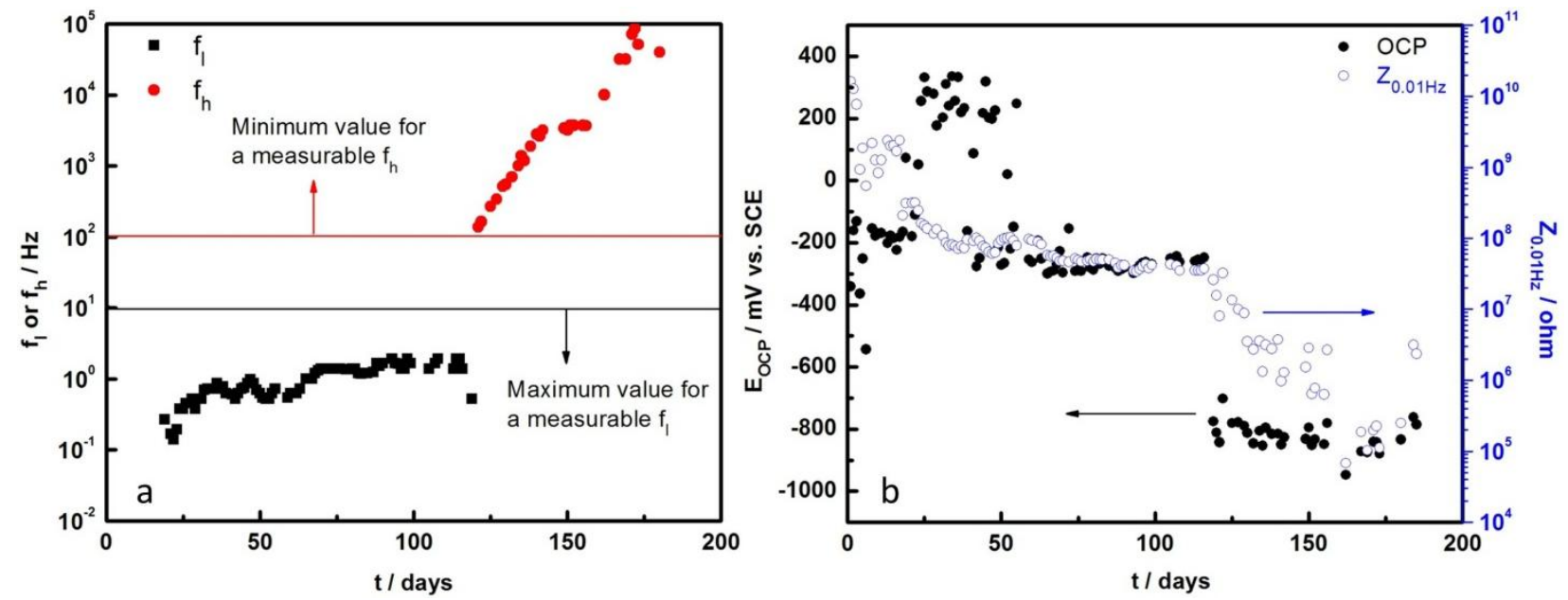

Fig. 4. Effect of exposure time on (a) the breakpoint frequency $f_{1}$ or $f_{h}$ and (b) OCP and $Z_{0.01 \mathrm{~Hz}}$ values. 


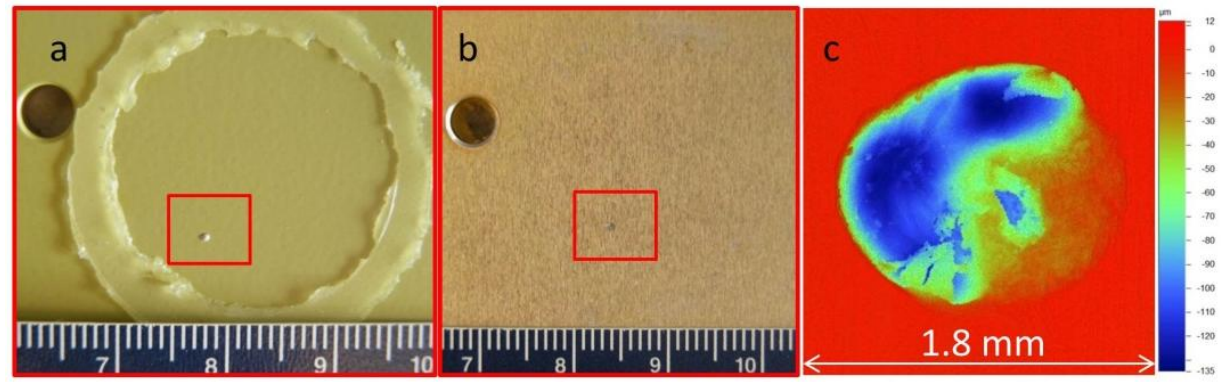

Fig. 5. Pitting corrosion mophology on Al alloy coated with CCC and chromate primer after 162 days EIS measurements. a. Before coating removed. b. After coating removed. c. Topography image by optical profilometry. 

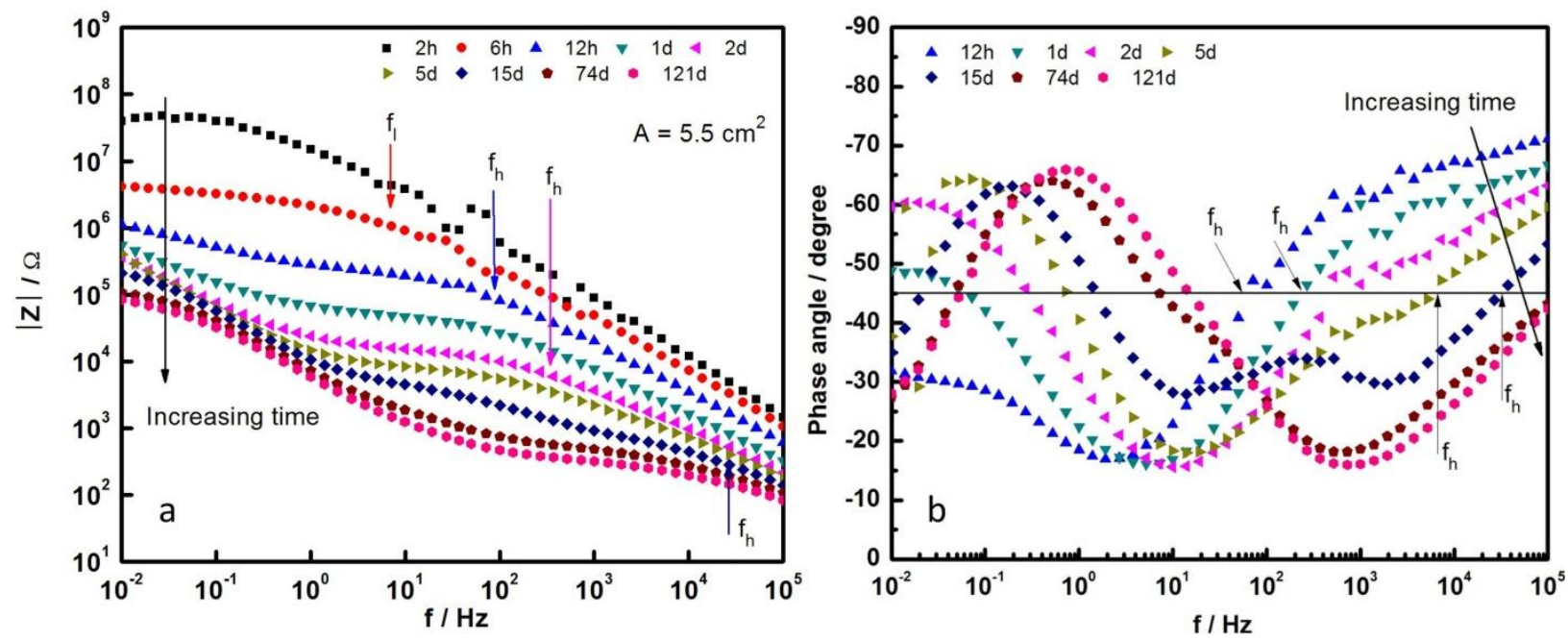

Fig. 6. Bode plots of $\mathrm{Al}$ alloy coated with adhesion promoter pretreatment and Pr-rich primer. EIS measurments were performed periodically during 121 days exposure to $5 \mathrm{wt} \% \mathrm{NaCl}$ solution. Sample area is $5.5 \mathrm{~cm}^{2}$. 

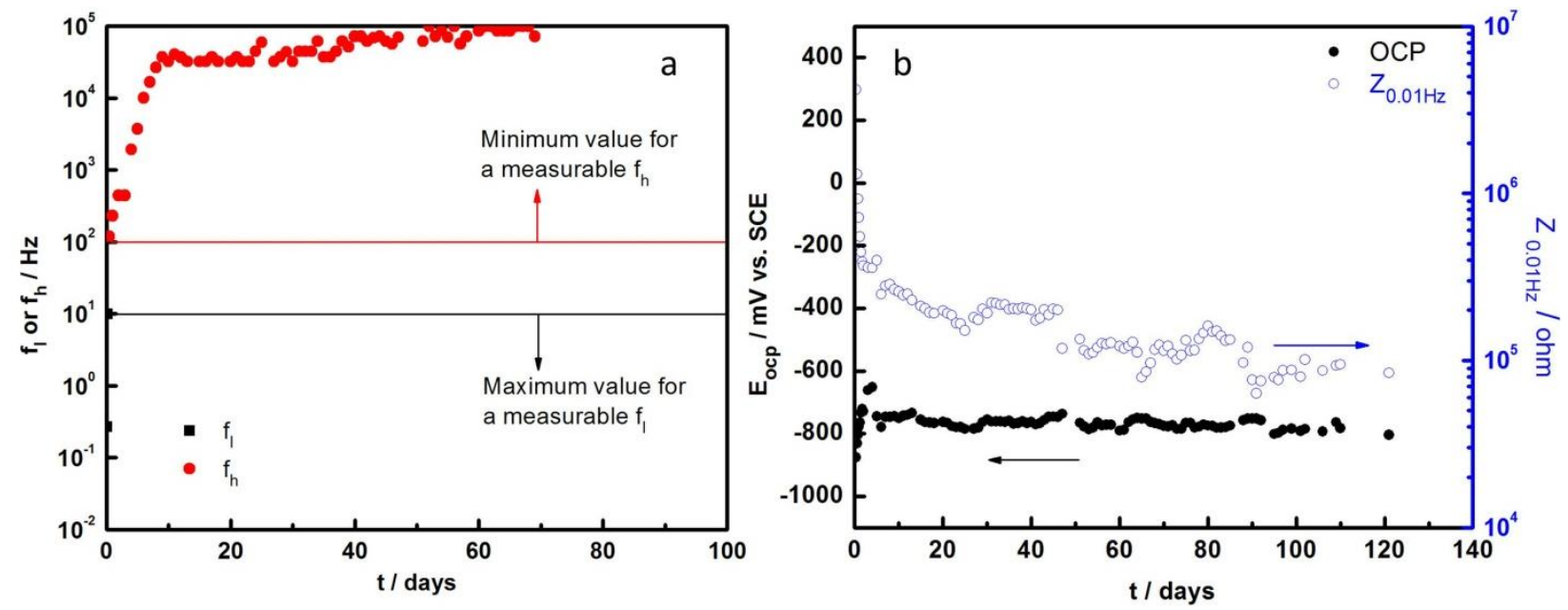

Fig. 7. Effect of exposure time on (a) breakpoint frequency $f_{1}$ or $f_{h}$ and (b) OCP and $Z_{0.01 \mathrm{~Hz}}$ values. The breakpoint frequency is higher than the measured frequency after 70 days. 

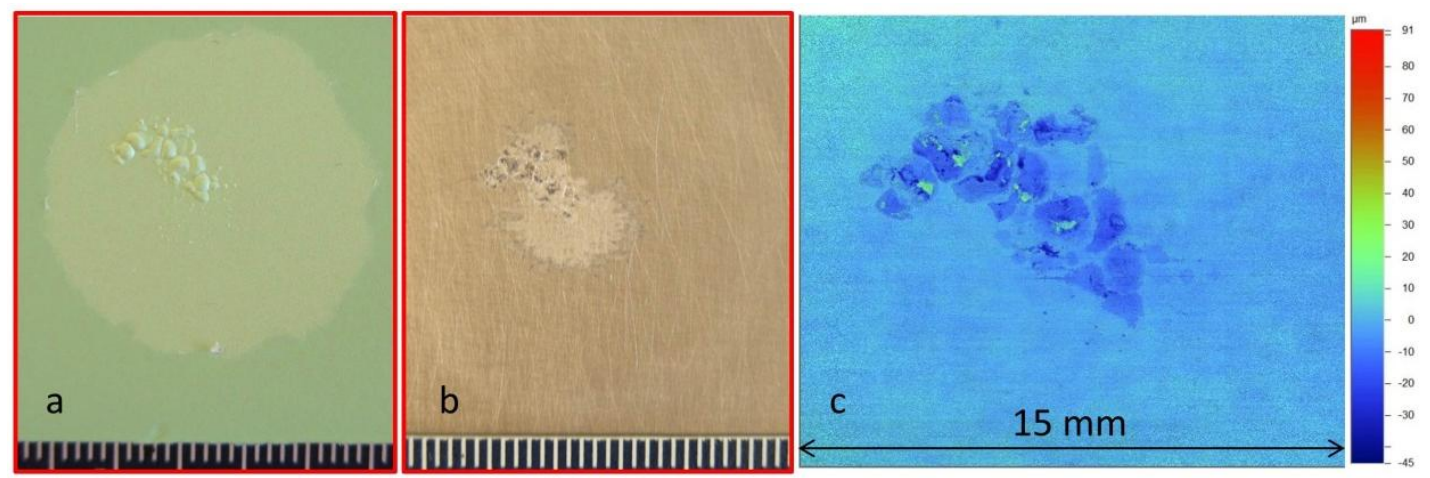

Fig. 8. Corrosion mophology on Al alloy coated with adhesion promoter pretreatment and Prrich primer after 121 days EIS measurements. a. Before coating removed. b. After coating removed. c. Topography image by optical profilometry. 

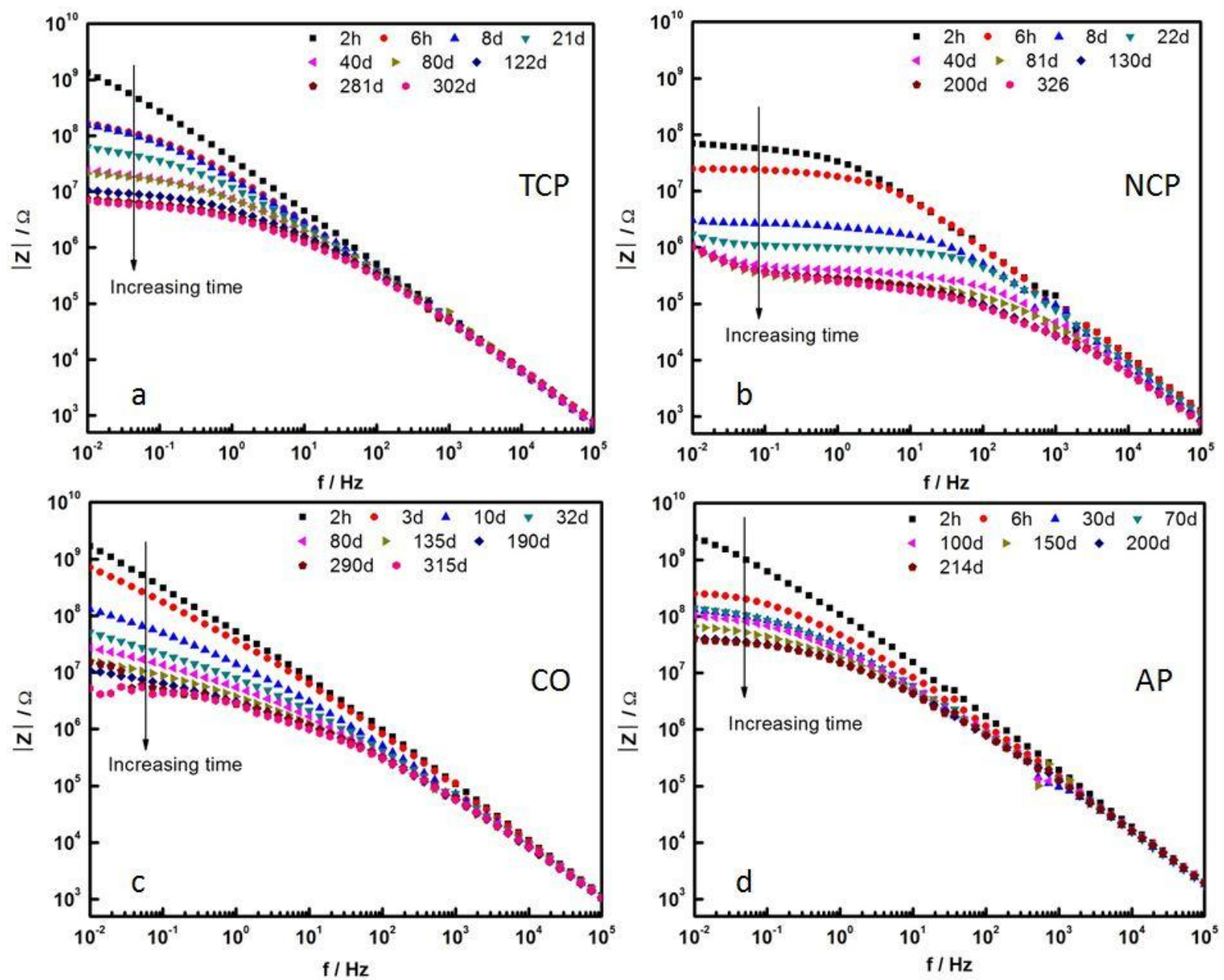

Fig. 9. Bode magnitude plots of Al alloy with (a) TCP pretreatment, (b) NCP pretreatment, (c) clean only, and (d) adhesion promoter pretreatment. They all have the same chromate primer after pretreatment. EIS measurments were performed periodically in $5 \mathrm{wt} \% \mathrm{NaCl}$ solution. Sample area is $5.5 \mathrm{~cm}^{2}$. 

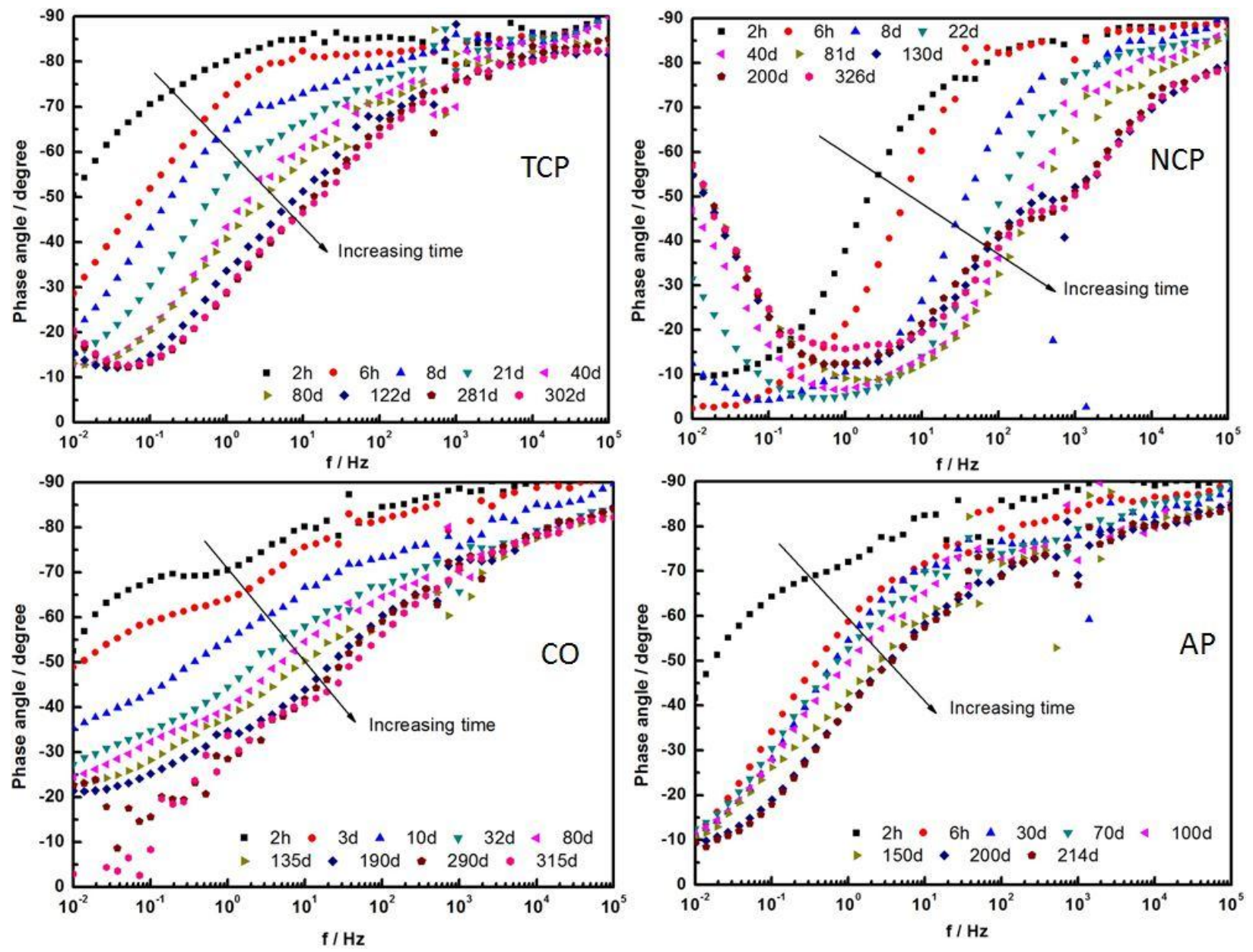

Fig. 10. Bode phase plots of Al alloy with (a) TCP pretreatment, (b) NCP pretreatment, (c) clean only, and (d) adhesion promoter pretreatment. They all have the same chromate primer after pretreatment. EIS measurments were performed periodically in $5 \mathrm{wt} \% \mathrm{NaCl}$ solution. Sample area is $5.5 \mathrm{~cm}^{2}$. 

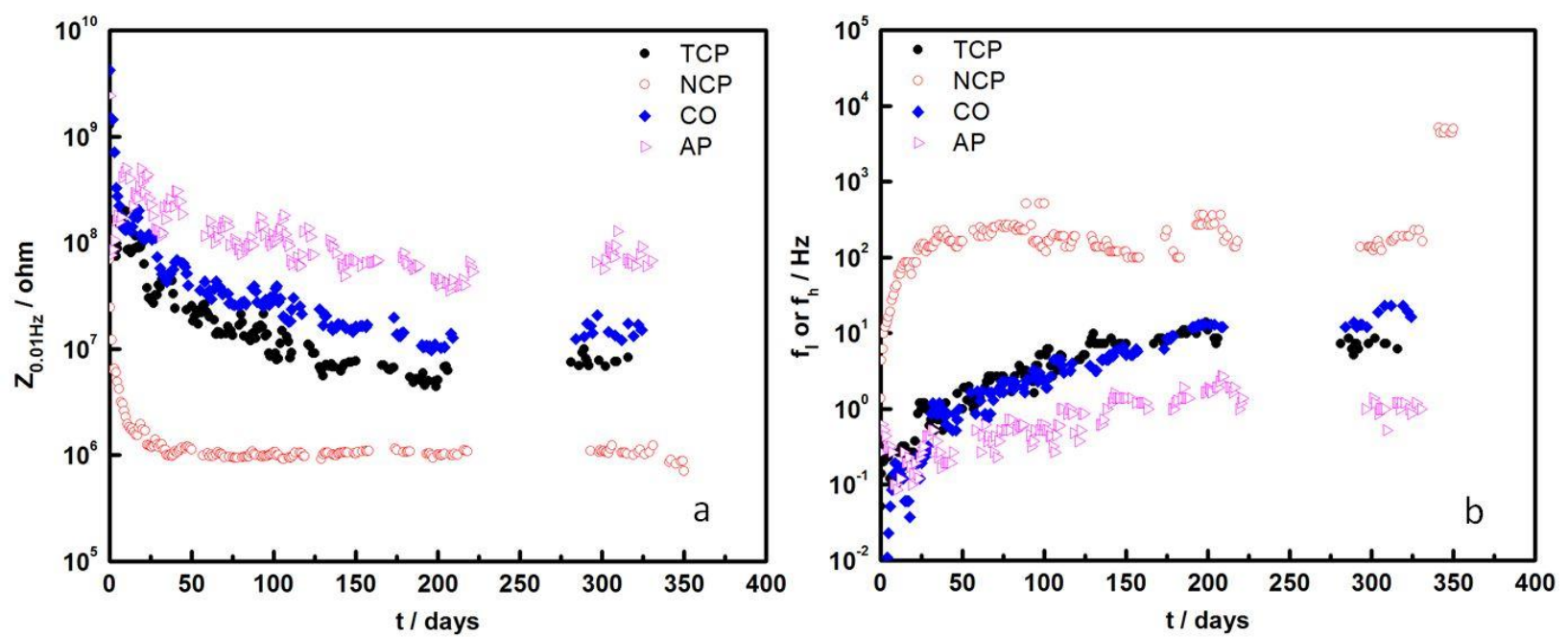

Fig. 11. Effect of exposure time on (a) $Z_{0.01 \mathrm{~Hz}}$ and (b) breakpoint frequency $\mathrm{f}_{1}$ and $\mathrm{f}_{\mathrm{h}}$ of different surface pretreatment samples. All the samples have the same chromate primer after pretreatment. Sample area is $5.5 \mathrm{~cm}^{2}$. 


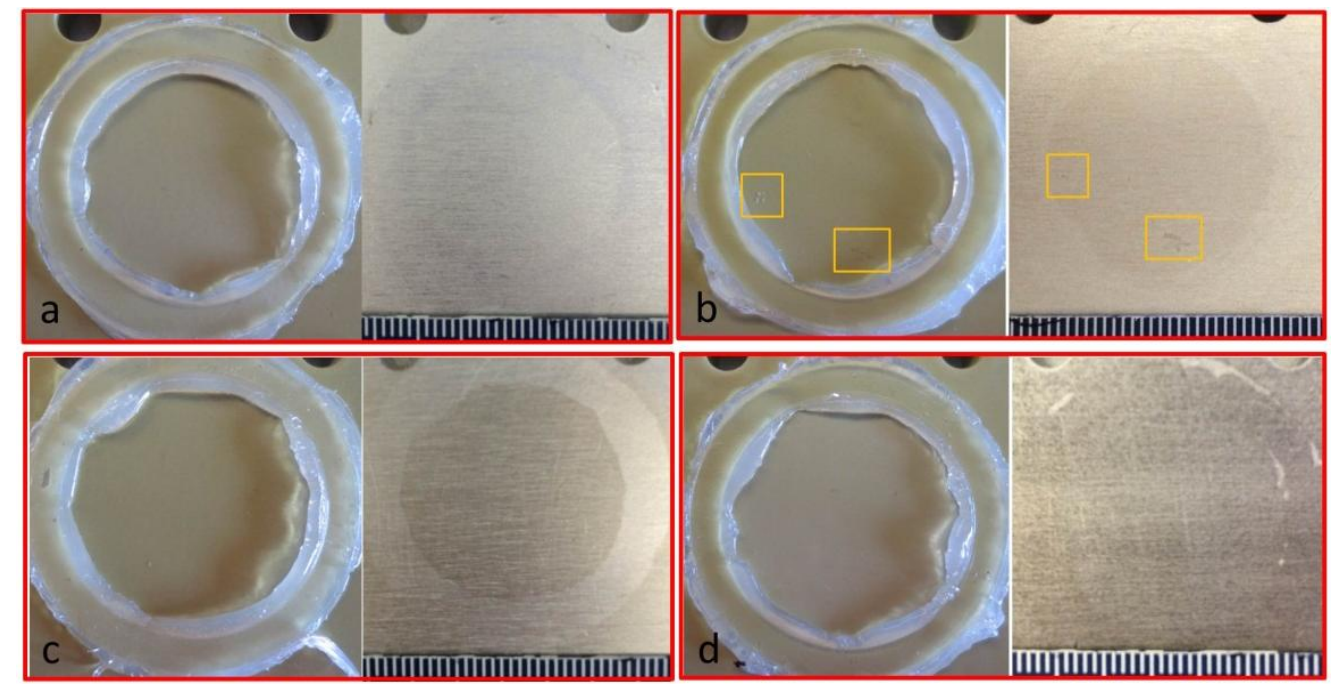

Fig. 12. Morphology after over 300 days exposure to $5 \mathrm{wt} \% \mathrm{NaCl}$ solution. (a) TCP, (b) NCP, (c) $\mathrm{CO}$, (d) AP. All the samples have the same chromate primer on the surface pretreatment. 


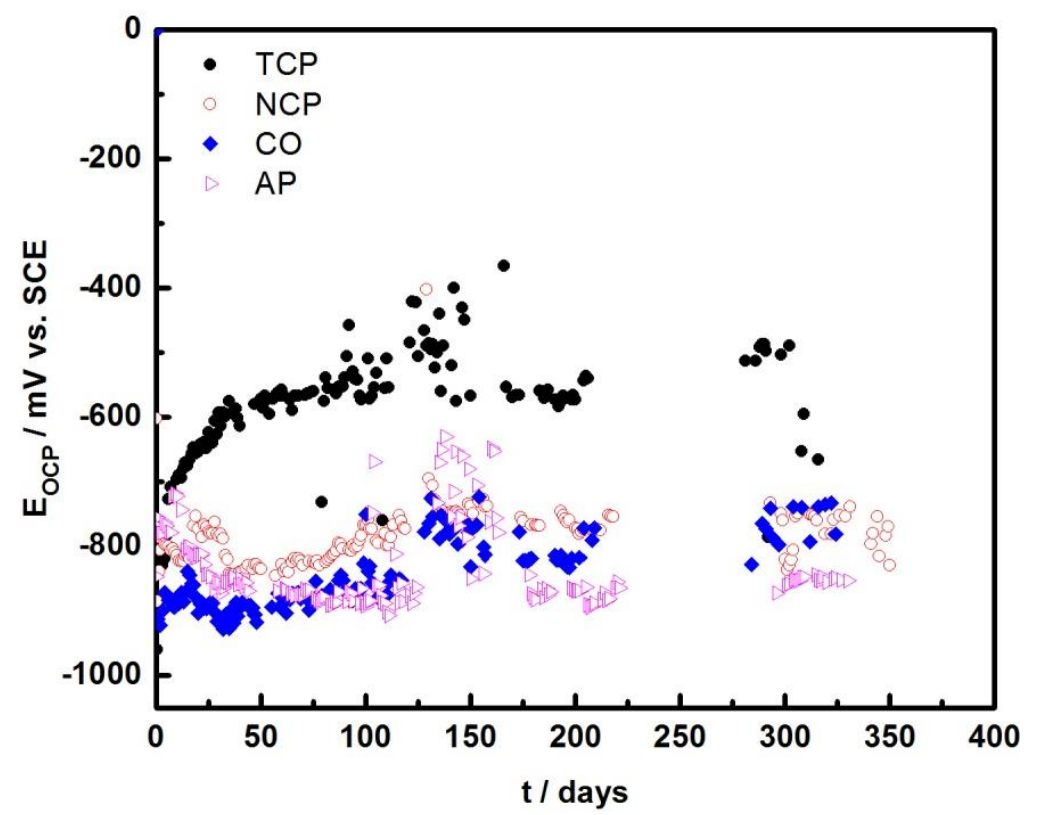

Fig. 13. OCP of different surface pretreatment samples changes as function of exposure time. All the samples have the same chromate primer after pretreatment. 

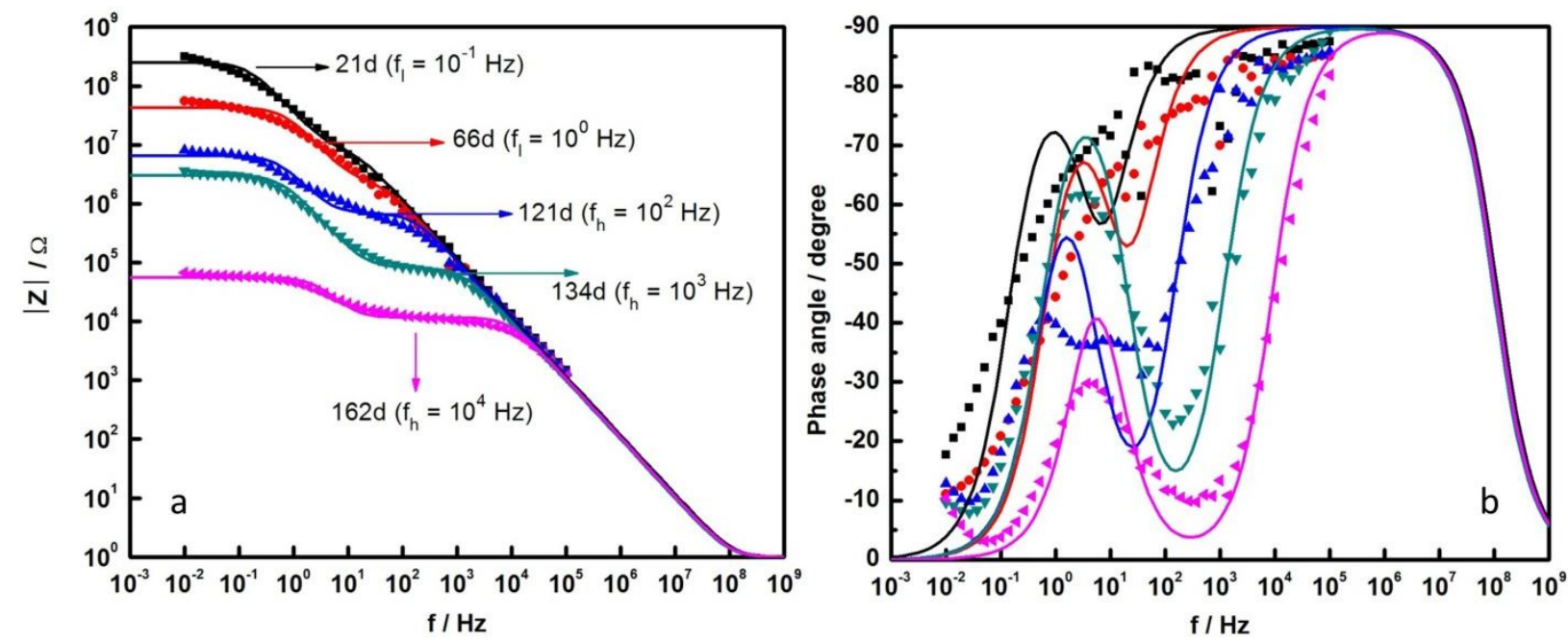

Fig. 14. The observed and fitted Bode plots of $\mathrm{Al}$ alloy coated with CCC pretreatment and chromate primer. The solid lines were fitted using the equivalent circuit in Fig. 1. Sample area is $5.5 \mathrm{~cm}^{2}$. 